\title{
SPARSE BOUNDS FOR THE DISCRETE SPHERICAL MAXIMAL FUNCTIONS
}

\author{
ROBERT KESLER, MICHAEL T. LACEY, AND DARÍO MENA
}

\begin{abstract}
We prove sparse bounds for the spherical maximal operator of Magyar, Stein and Wainger. The bounds are conjecturally sharp, and contain an endpoint estimate. The new method of proof is inspired by ones by Bourgain and lonescu, is very efficient, and has not been used in the proof of sparse bounds before. The HardyLittlewood Circle method is used to decompose the multiplier into major and minor arc components. The efficiency arises as one only needs a single estimate on each element of the decomposition.
\end{abstract}

\section{Contents}

1. Introduction 1

2. Proof of the Sparse Bounds inside the polygon $Z_{d}$

3. The Endpoint Sparse Bound 14

4. Interpolation of Sparse Bounds 16

5. Counterexamples 18

$\begin{array}{ll}\text { References } & 19\end{array}$

\section{INTRODUCTION}

Let $\mathcal{A}_{\lambda} \mathrm{f}=\mathrm{d} \sigma_{\lambda} * \mathrm{f}$ where $\mathrm{d} \sigma_{\lambda}$ is a uniform unit mass spherical measure on a sphere of radius $\lambda$ in $\mathbb{R}^{d}$, for $d \geq 3$. Set the Stein spherical maximal operator to be

$$
\mathcal{A f}(\mathrm{x})=\sup _{\lambda>0} \mathcal{A}_{\lambda} \mathrm{f},
$$

where $f$ is a non-negative compactly supported and bounded function. We are interested in sparse bound for the maximal function. In the continuous case, this estimate holds, and is sharp, up to the boundary.

Research supported in part by grant from the US National Science Foundation, DMS-1600693 and the Australian Research Council ARC DP160100153.

Research supported by project 821-B8-287, CIMPA, Escuela de Matemática, UCR.. 


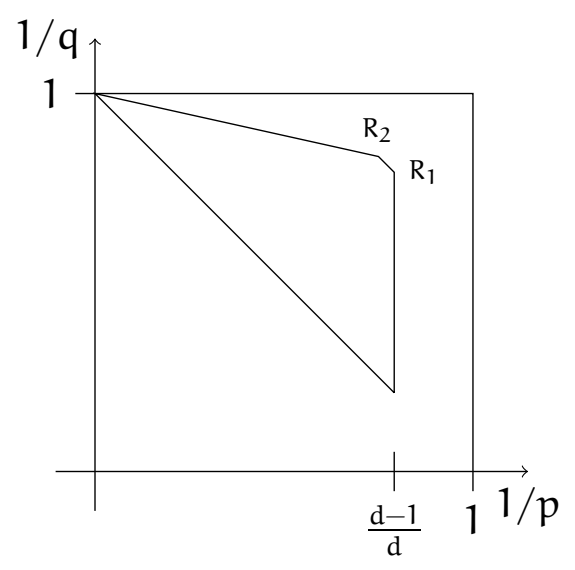

FiguRE 1. Sparse bounds hold for points $(1 / p, 1 / q)$ in the interior of the four sided region $R_{d}$. The point $R_{1}$ is $\left(\frac{d-1}{d}, \frac{d-1}{d}\right)$ and $R_{2}$ is $\left(\frac{d^{2}-d}{d^{2}+1}, \frac{d^{2}-d+2}{d^{2}+1}\right)$.

Theorem 1.1. [11] Let $\mathrm{d} \geq 3$ and set $\mathbf{R}_{\mathrm{d}}$ to be the polygon with vertices $\mathrm{R}_{0}=\left(\frac{\mathrm{d}-1}{\mathrm{~d}}, \frac{1}{\mathrm{~d}}\right)$, $\mathrm{R}_{1}=\left(\frac{\mathrm{d}-1}{\mathrm{~d}}, \frac{\mathrm{d}-1}{\mathrm{~d}}\right), \mathrm{R}_{2}=\left(\frac{\mathrm{d}^{2}-\mathrm{d}}{\mathrm{d}^{2}+1}, \frac{\mathrm{d}^{2}-\mathrm{d}+2}{\mathrm{~d}^{2}+1}\right)$, and $\mathrm{R}_{3}=(0,1)$. (See Figure 1.) Then, for all $\left(\frac{1}{p}, \frac{1}{q}\right)$ in the interior $\mathbf{R}_{d}$, we have the sparse bound $\|\mathcal{A}\|_{p, q}<\infty$.

We set notation for the sparse bounds. Call a collection of cubes $\mathcal{S}$ in $\mathbb{R}^{n}$ sparse if there are sets $\left\{E_{S}: S \in \mathcal{S}\right\}$ which are pairwise disjoint, $E_{S} \subset S$ and satisfy $\left|E_{S}\right|>\frac{1}{4}|S|$. For any cube $\mathrm{Q}$ and $1 \leq \mathrm{r}<\infty$, set $\langle\mathrm{f}\rangle_{\mathrm{Q}, \mathrm{r}}^{\mathrm{r}}=|\mathrm{Q}|^{-1} \int_{\mathrm{Q}}|\mathrm{f}|^{\mathrm{r}} \mathrm{d} x$. Then the $(\mathrm{r}, \mathrm{s})$-sparse form $\Lambda_{\mathcal{S}, r, s}=\Lambda_{r, s}$, indexed by the sparse collection $\mathcal{S}$ is

$$
\Lambda_{S, r, s}(f, g)=\sum_{S \in \mathcal{S}}|S|\langle f\rangle_{S, r}\langle g\rangle_{S, s}
$$

For a sublinear operator $\mathrm{T}$, we set $\|\mathrm{T}\|_{\mathrm{r}, \mathrm{s}}$ to be the best constant $\mathrm{C}$ in the inequality

$$
\langle\mathrm{Tf}, \mathrm{g}\rangle<\mathrm{C} \sup _{\mathcal{S}} \Lambda_{\mathrm{S}, \mathrm{r}, \mathrm{s}}(\mathrm{f}, \mathrm{g})
$$

We use the same notation for sublinear operators $T$ acting on functions defined on $\mathbb{Z}^{d}$.

The theorem above refines the well-known $\mathrm{L}^{\mathrm{p}}$-improving properties for the local maximal function $\sup _{1 \leq \lambda \leq 2} \mathcal{A}_{\lambda} * \mathrm{f}$, proved by Schlag [15] and Schlag and Sogge [16]. Also see [12]. The Theorem above has as immediate corollaries (a) vector valued inequalities, and (b) weighted consequences. Both sets of consequences are the strongest known. The method of proof uses the $\mathrm{L}^{\mathrm{p}}$-improving inequalities for the spherical maximal function. That is, the proof is, in some sense, standard, although only recently discovered, and yields the best known information about the mapping properties of the spherical maximal function. 
We turn to the setting of discrete spherical averages. Provided $\lambda^{2}$ is an integer, and dimension $d \geq 5$, we can define

$$
A_{\lambda} f(x)=\lambda^{2-d} \sum_{n \in \mathbb{Z}^{d}:|n|=\lambda} f(x-n)
$$

for functions $f \in \ell^{2}\left(\mathbb{Z}^{\mathrm{d}}\right)$. We restrict attention to the case of $d \geq 5$ as in that case for all $\lambda^{2} \in \mathbb{N}$, the cardinality of $\left\{n \in \mathbb{Z}^{\mathrm{d}}:|n|=\lambda\right\} \simeq \lambda^{\mathrm{d}-2}$. Let $A f=\sup _{\lambda} A_{\lambda} \mathrm{f}$, where we will always understand that $\lambda^{2} \in \mathbb{N}$. This is the maximal function of Magyar [14] and Magyar, Stein and Wainger [13]. The following Theorem is the best known extension of the sparse bounds for the continuous spherical maximal function to the discrete setting.

Theorem 1.2. Let $\mathrm{Z}_{\mathrm{d}}$ be the polygon with vertices

$$
Z_{j}=\frac{d-4}{d-2} R_{j}+\frac{2}{d-2}\left(\frac{1}{2}, \frac{1}{2}\right), \quad j=0,1,2,
$$

and $Z_{3}=(0,1)$. (See Figure 2.) There holds:

(1) For all $\left(\frac{1}{p}, \frac{1}{q}\right)$ in the interior $\mathbf{Z}_{\mathrm{d}}$, we have the sparse bound $\|A f\|_{p, q}<\infty$.

(2) With $\mathrm{f}=\mathbf{1}_{\mathrm{F}}$ and $\mathrm{g}=\mathbf{1}_{\mathrm{G}}$, there holds

$$
\left\langle A \mathbf{1}_{\mathrm{F}}, \mathbf{1}_{\mathrm{G}}\right\rangle \lesssim \sup _{\mathcal{S}} \Lambda_{\mathcal{S}, \frac{\mathrm{d}}{\mathrm{d}-2}, \frac{\mathrm{d}}{\mathrm{d}-2}}\left(\mathbf{1}_{\mathrm{F}}, \mathbf{1}_{\mathrm{G}}\right) .
$$

By direct computation, $Z_{0}=\left(\frac{d-2}{d}, \frac{2}{d}\right), Z_{1}=\left(\frac{d-2}{d}, \frac{d-2}{d}\right)$ and

$$
Z_{2}=\left(\frac{d^{3}-4 d^{2}+4 d+1}{d^{3}-2 d^{2}+d-2}, \frac{d^{3}-4 d^{2}+6 d-7}{d^{3}-2 d^{2}+d-2}\right) .
$$

The sparse bound near the point $\left(\frac{d-2}{d}, \frac{2}{d}\right)$ implies the maximal inequality of Magyar, Stein and Wainger [13], namely that $A: \ell^{\mathfrak{p}}\left(\mathbb{Z}^{\mathrm{d}}\right) \rightarrow \ell^{\mathfrak{p}}\left(\mathbb{Z}^{\mathrm{d}}\right)$, for $p>\frac{d}{d-2}$. The sparse bound (1.4) requires that both functions be indicator sets, and so is of restricted weak type. It implies the restricted weak type inequality of lonescu [6]. These inequalities imply a wide range of weighted and vector valued inequalities, all of which are new. See the applications of the sparse bound in the continuous case in [11].

The discrete spherical maximal function $\ell^{p}$ bounds were established by Magyar, Stein and Wainger [13], with an endpoint restricted weak-type estimate proved by lonescu [6]. The discrete $\ell^{p}$-improving inequalities have only recently been investigated. The case of a fixed radius was addressed, independently, in $[5,9]$. Spherical maximal functions, restricting to lacunary and super lacunary cases require different techniques $[3,4,10]$. Robert Kesler established sparse bounds for the discrete case in $[7,8]$. This paper extends and simplifies those arguments.

It is very tempting to conjecture that our sparse bounds form the sharp range, up to the endpoints. One would expect that certain kinds of natural examples would demonstrate this. But examples are much harder to come by in the discrete setting. We return to this in $\S 5$.

The argument in this paper is elegant, especially if one restricts attention to the endpoint estimate (1.4). And much simpler than the arguments in $[7,8]$. It proceeds by 


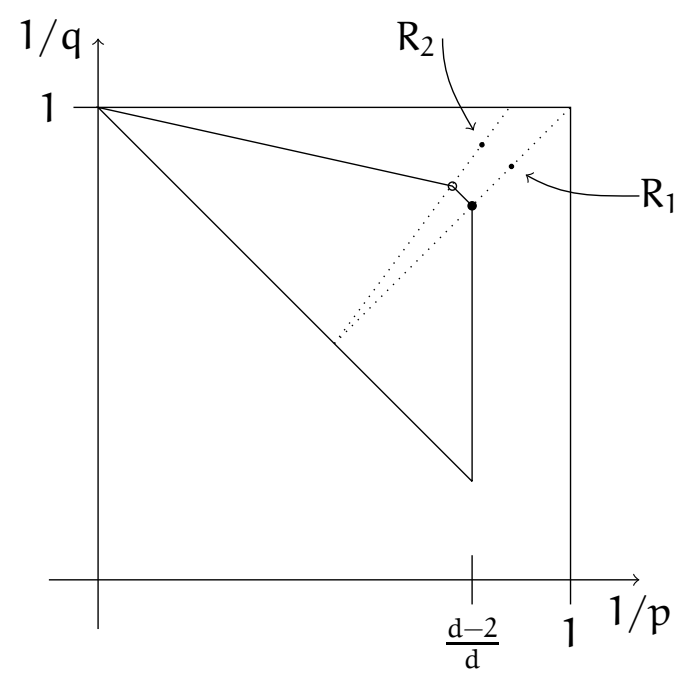

FiguRE 2. Sparse bounds for the discrete spherical maximal function hold for points $(1 / p, 1 / q)$ in the interior of the four sided figure above. The dotted lines pass through the points $(1 / 2,1 / 2)$ and the points $R_{1}$ and $R_{2}$ of Figure 1. Circles along these lines are the points $Z_{1}$ and $Z_{2}$. The restricted weak-type sparse bound (1.4) holds at the filled in circle, $Z_{1}=\left(\frac{d}{d-2}, \frac{d}{d-2}\right)$.

decomposing the maximal function into a series of terms, guided by the Hardy-Littlewood circle method decomposition developed by Magyar, Stein and Wainger [13]. The decomposition has many parts, as indicated in Figure 3 and Figure 4. But, for each part of the decomposition, we need only one estimate, either an $\ell^{2}$ estimate, or an endpoint estimate. Roughly speaking, one uses either a 'high frequency' $\ell^{2}$ estimate, or a 'low frequency' inequality, in which one compares to smoother averages. Interestingly, the notion of 'smoother averages' varies. The argument of lonescu combined with the sparse perspective yields a powerful inequality. Notations and conventions will be established in this section, and used throughout the paper.

\section{Proof of the Sparse Bounds inside the polygon $\mathbf{Z}_{\mathrm{d}}$}

A sparse bound is typically proved by recursion. So, the main step is to prove the recursive statement. To do this, we fix a large dyadic cube $E$, functions $f=\mathbf{1}_{\mathrm{F}}$ and $g=1_{G}$ supported on $E$. We say that $\tau: E \rightarrow\{1, \ldots, \ell E\}$ is an admissible stopping time if for any subcube $Q \subset E$ with $\langle f\rangle_{Q}>C\langle f\rangle_{E}$, for some large constant $C$ to be chosen later, we have $\min _{x \in Q} \tau(x)>\ell Q$.

Lemma 2.1. Let $\left(\frac{1}{\mathrm{p}}, \frac{1}{\mathrm{q}}\right)$ be in the interior of $\mathbf{Z}_{\mathrm{d}}$. For any dyadic cube, functions $f=\mathbf{1}_{\mathrm{F}}$ and $\mathrm{g}=\mathbf{1}_{\mathrm{G}}$ supported on $\mathrm{E}$, and any admissible stopping time $\tau$, there holds

$$
|E|^{-1}\left\langle A_{\tau} f, g\right\rangle \lesssim\langle f\rangle_{E}^{1 / p}\langle g\rangle_{E}^{1 / q}
$$


We complete the proof of the main result.

Proof of First Part of Theorem 1.2. We can assume that there is a fixed dyadic cube $E$ so that $f=1_{F}$ is supported on cube $3 E$, and $g=1_{G}$ is supported on $E$. Let $\mathcal{Q}_{\mathrm{E}}$ be the maximal dyadic subcubes of $E$ for which $\langle f\rangle_{3 Q}>C\langle f\rangle_{3 E}$, for a large constant $C$. Observe that we have, for an appropriate choice of admissible $\tau(x)$,

$$
\left\langle\sup _{\lambda \leq \ell(E)} A_{\lambda} f, g\right\rangle \leq\left\langle A_{\tau} f, g\right\rangle+\sum_{Q \in \mathcal{Q}_{E}}\left\langle\sup _{\lambda \leq \ell(Q)} A_{\lambda}\left(f \mathbf{1}_{3 Q}\right), g \mathbf{1}_{Q}\right\rangle .
$$

The first term is controlled by (2.2). For appropriate constant $C \simeq 3^{d}$, we have

$$
\sum_{\mathrm{Q} \in \mathcal{Q}_{\mathrm{E}}}|\mathrm{Q}| \leq \frac{1}{4}|\mathrm{E}|
$$

We can clearly recurse on the second term above to construct our sparse bound. This proves a sparse bound for all indicator functions in the interior of $\mathbf{Z}_{\mathrm{d}}$.

Sparse bounds for indicator functions in an open set self-improve to sparse bounds for functions. We give the details in the last section, see Lemma 4.1.

We use the corresponding recursive inequality for spherical averages on $\mathbb{R}^{\mathrm{d}}$. Recall that $\mathcal{A}_{\lambda}$ is the continuous spherical average.

Lemma 2.3. [11, Lemma 3.4] Let $\left(\frac{1}{\bar{p}}, \frac{1}{\bar{q}}\right)$ be in the interior of $\mathbf{R}_{\mathrm{d}}$. For any dyadic cube $\mathrm{E}$, functions $\phi=1_{\mathrm{F}}$ and $\gamma=\mathbf{1}_{\mathrm{G}}$ supported on $\mathrm{E}$, and any admissible stopping time $\tau$, there holds

$$
|\mathrm{E}|^{-1}\left\langle\mathcal{A}_{\tau} \phi, \gamma\right\rangle \lesssim\langle\phi\rangle_{\mathrm{E}}^{1 / \overline{\mathrm{p}}}\langle\gamma\rangle_{\mathrm{E}}^{1 / \overline{\mathrm{q}}} \text {. }
$$

We turn to the proof of Lemma 2.1. The restriction to indicator functions will allow us to use interpolation arguments, even though our setting has stopping times, and hence is non-linear. Let $L$ be the line through $\left(\frac{1}{2}, \frac{1}{2}\right)$ and $\left(\frac{1}{p}, \frac{1}{q}\right)$. Then, let $\left(\frac{1}{\bar{p}}, \frac{1}{\bar{q}}\right)$ be a point on $L$ that is in the interior of $\mathbf{R}_{d}$, and very close to the boundary. (The dashed lines in Figure 2 are examples of the lines $L$ we are discussing here.)

This is the point: Fix $(1 / \bar{p}, 1 / \bar{q}) \in \mathbf{R}_{d}$. For all sufficiently small $0<\epsilon<1$ so that $(1 /(\bar{p}+\epsilon), 1 /(\bar{q}+\epsilon)) \in R_{d}$, and integers $N \in \mathbb{N}$, we can write $A_{\tau} f \leq M_{1}+M_{2}$ where

$$
\begin{aligned}
& |E|^{-1}\left\langle M_{1}, g\right\rangle \lesssim N^{1+\epsilon}\langle f\rangle_{E}^{\frac{1}{\bar{p}+\epsilon}}\langle g\rangle_{E}^{\frac{1}{\bar{q}+\epsilon}}, \\
& |E|^{-1}\left\langle M_{2}, g\right\rangle \lesssim N^{d \epsilon+\frac{4-d}{2}}\langle f\rangle_{E}^{1 / 2}\langle g\rangle_{E}^{1 / 2} .
\end{aligned}
$$

Implied constants depend upon $\bar{p}, \bar{q}$ and $\epsilon$, but we do not track the dependence. Once this is proved, one has

$$
|E|^{-1}\left\langle A_{\tau} f, g\right\rangle \lesssim N^{1+\epsilon}\langle f\rangle_{E}^{\frac{1}{\bar{p}+\epsilon}}\langle g\rangle_{E}^{\frac{1}{\bar{q}+\epsilon}}+N^{d \epsilon+\frac{4-d}{2}}\langle f\rangle_{E}^{1 / 2}\langle g\rangle_{E}^{1 / 2}
$$


Choosing $N$ to minimize the right hand side, and letting $(1 / \bar{p}, 1 / \bar{q})$ and $0<\epsilon<1$ vary completes the proof. Indeed, ignoring $\epsilon$ 's, we see that the value of $p$ is given by

$$
\frac{1}{p}=\frac{1}{\bar{p}}+\frac{2}{d-2}\left(\frac{1}{2}-\frac{1}{\bar{p}}\right)=\frac{2}{d-2} \cdot \frac{1}{2}+\frac{d-4}{d-2} \cdot \frac{1}{\bar{p}}
$$

Compare this to our description of the extreme points of $\mathbf{Z}_{\mathrm{d}}$ in (1.3). Thus, our Lemma follows.

In proving (2.5) and (2.6), it suffices, given $0<\epsilon<1$, to prove the statement for sufficiently large $N$. We will do so for $N>N_{0}$, for a sufficiently large choice of $N_{0}>0$. Indeed, we find it necessary to use an absorption argument. We show that

$$
A_{\tau} f \leq M_{1}+M_{2}+\frac{1}{2} A_{\tau} f
$$

where $M_{1}$ and $M_{2}$ are as in (2.5) and (2.6).

The reader can consult Figure 3 for a guide to the argument. For a technical reason, we assume that $F \subset\left(2 \mathbb{Z}^{\mathrm{d}}\right)+\delta_{f}$, and $\mathrm{G} \subset\left(2 \mathbb{Z}^{\mathrm{d}}\right)+\delta_{g}$. Here, $\delta_{f}, \delta_{g} \in\{0,1\}^{\mathrm{d}}$. This can be assumed without loss of generality.

Small values of $\tau$. The terms $M_{1}$ and $M_{2}$ have several components. The first contribution to $M_{1}$ is the term $M_{1,1}=\mathbf{1}_{\tau \leq N^{1+\varepsilon}} A_{\tau} f$. Our verification that $M_{1,1}$ satisfies (2.5) is our first application of the $\mathbb{R}^{\mathrm{d}}$ inequality (2.4).

We need functions on $\mathbb{R}^{\mathrm{d}}$. Take $\phi(x)=\sum_{n \in \mathbb{Z}^{\mathrm{d}}} \mathbf{1}_{\mathrm{F}}(\mathrm{n}) \mathbf{1}_{\mathrm{n}+[-1,1)^{\mathrm{d}}}(x)$, and define $\gamma$ similarly. By the reduction we made above, these are indicator functions. Moreover, if $\tau$ is admissible stopping time for $f$, then it is for $\phi$ as well. The inequality (2.4) holds for these two functions on $\mathbb{R}^{d}$. Then, notice that we can compare the discrete and continuous spherical averages as follows.

$$
A_{\tau} f(x) \lesssim \tau \mathcal{A}_{\tau} \phi(x) .
$$

Therefore, if we require that $\tau \leq N^{1+\epsilon}$, we see that (2.4) implies that $M_{1,1}$ satisfies (2.5).

The Decomposition. Below, we assume that $\tau>N^{1+\epsilon}$ pointwise. At this point, we need a decomposition of $A_{\lambda} f$ into a family of multipliers. We recall this from Magyar, Stein and Wainger [13]. Upper case letters denote a convolution operator, and lower case letters denote the corresponding multiplier. Let $e(x)=e^{2 \pi i x}$ and for integers $q, e_{q}(x)=e(x / q)$.

$$
\begin{aligned}
A_{\lambda} f & =C_{\lambda} f+E_{\lambda} f \\
C_{\lambda} f & =\sum_{1 \leq q \leq \lambda} \sum_{a \in \mathbb{Z}_{q}^{x}} e_{q}\left(-\lambda^{2} a\right) C_{\lambda}^{a / q} f, \\
c_{\lambda}^{a / q}(\xi) & =\widehat{C_{\lambda}^{a / q}}(\xi)=\sum_{\ell \in \mathbb{Z}_{q}^{d}} G(a / q, \ell) \widetilde{\psi}_{q}(\xi-\ell / q) \widetilde{d \sigma_{\lambda}}(\xi-\ell / q), \\
G(a / q, \ell) & =q^{-d} \sum_{n \in \mathbb{Z}_{q}^{d}} e_{q}\left(|n|^{2} a+n \cdot \ell\right) .
\end{aligned}
$$




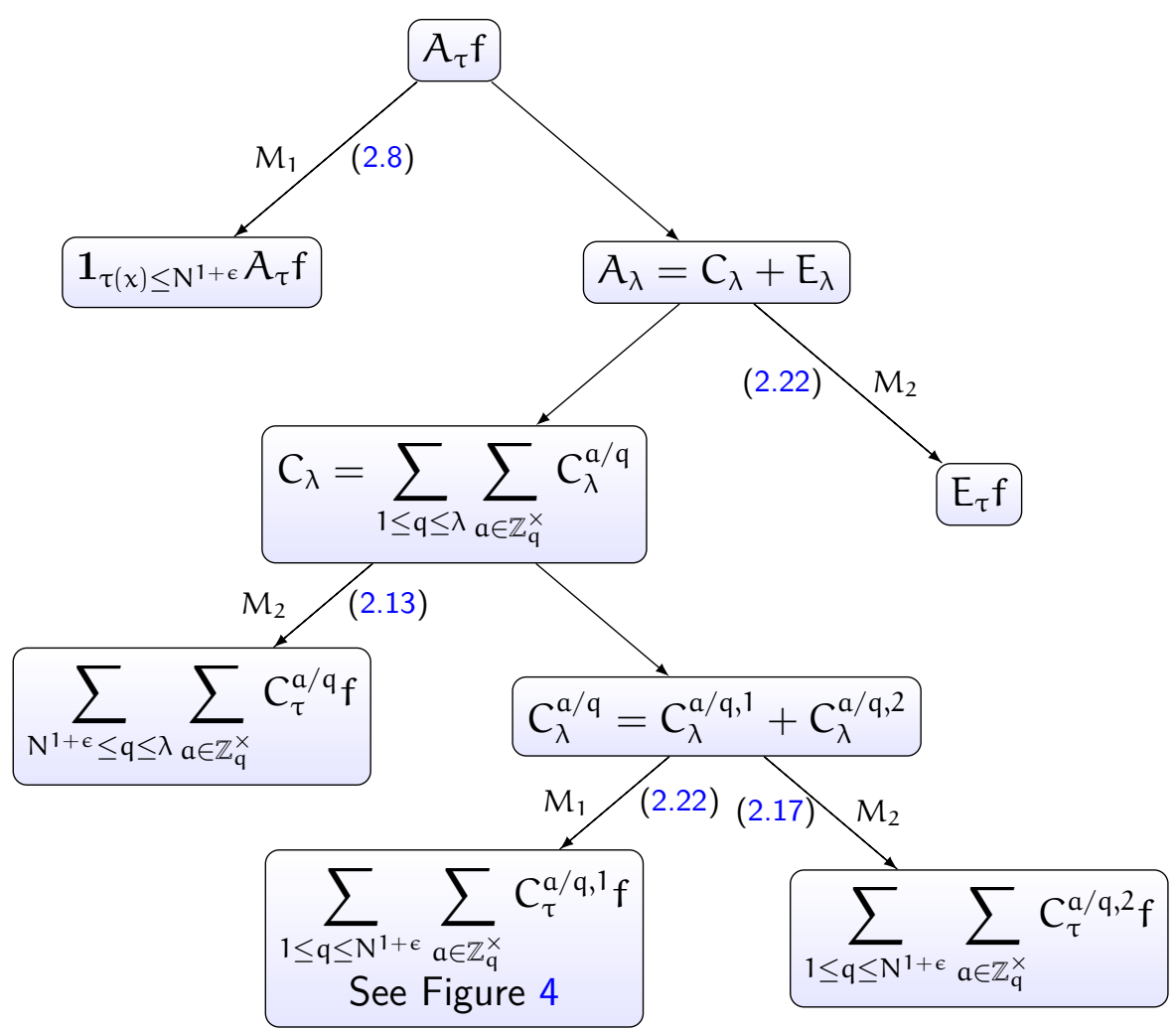

FiguRE 3. The flow of the proof of (2.2). The nodes of the tree indicate the different elements of the decomposition, and a label on an arrow shows which of $M_{1}$ or $M_{2}$ that term contributes to. Above, $\lambda$ represents a fixed choice of radius, and $\tau=\tau(x)$ an admissible choice of radius. For space considerations, several terms of the form $e_{q}\left(-\lambda^{2} a\right)$ have been omitted, compare to $(2.10)$.

The term $G(a / q, \ell)$ is a normalized Gauss sum. In (2.10), the sum over $a \in \mathbb{Z}_{\mathrm{q}}^{\times}$means that $(a, q)=1$. In $(2.11)$, the hat indicates the Fourier transform on $\mathbb{Z}^{\mathrm{d}}$, and the notation conflates the operator $C_{\lambda}^{a / q}$, and the kernel. All our operators are convolution operators or maximal operators formed from the same. The function $\psi$ is a Schwartz function on $\mathbb{R}^{\mathrm{d}}$ which satisfies

$$
\mathbf{1}_{\left[-\frac{1}{2}, \frac{1}{2}\right]}(|\xi|) \leq \widetilde{\psi}(\xi) \leq \mathbf{1}_{[-1,1]}(|\xi|) \text {. }
$$

Above, $\tilde{f}$ denotes the Fourier transform of $f$ on $\mathbb{R}^{d}$, and $\widetilde{\psi}_{q}(\xi)=\widetilde{\psi}(q \xi)$. The Fourier transform on $\mathbb{R}^{\mathrm{d}}$ of $d \sigma_{\lambda}$ is $\widetilde{\mathrm{d} \sigma_{\lambda}}$. Finally, we will use the notation $\lambda$ for describing multipliers and so on, and using $\tau$ especially when obtaining estimates. In this way, many supremums will be suppressed from the notation. 
The Error Term $E_{\lambda}$. The first contribution to $M_{2}$ is $M_{2,1}=\left|E_{\tau} f\right|$. The inequality below is from [13, Prop. 4.1], and it implies that $M_{2,1}$ satisfies (2.6) since $\tau>N^{1+\epsilon}$.

$$
\left\|\sup _{\Lambda \leq \lambda \leq 2 \Lambda} \mid E_{\lambda} \cdot\right\|_{2 \rightarrow 2} \lesssim \Lambda^{\frac{4-\mathrm{d}}{2}}, \quad \Lambda \geq 1 .
$$

Large Denominators. The second contribution to $\mathrm{M}_{2}$ is

$$
M_{2,2}=\left|\sum_{N^{1+e} \leq q \leq \tau} e_{q}\left(-\lambda^{2} a\right) C_{\tau}^{a / q} f\right|
$$

The estimate below is a result of Magyar, Stein and Wainger [13, Prop. 3.1], and it verifies that $M_{2,2}$ satisfies (2.6). We need only sum it over $1 \leq a \leq q$, and $q>N^{1+\epsilon}$.

$$
\left\|\sup _{\lambda>\mathrm{q}}\left|C_{\lambda}^{a / q} f\right|\right\|_{2} \lesssim q^{-\frac{d}{2}}\|f\|_{2} .
$$

Small Denominators: A Secondary Decomposition. It remains to bound the small denominator case, namely

$$
\sum_{1 \leq q \leq N^{1}+\varepsilon} \sum_{a \in \mathbb{Z}_{q}^{\times}} e_{q}\left(-\lambda^{2} a\right) C_{\tau}^{a / q} f
$$

with further contributions to $M_{1}$ and $M_{2}$. Write $C_{\tau}^{a / q}=C_{\tau}^{a / q, 1}+C_{\tau}^{a / q, 2}$, with this understanding. For an integer $1 \leq \mathrm{Q} \leq \mathrm{N} / 2$, and $\mathrm{Q} \leq \mathrm{q}<2 \mathrm{Q}$, define

$$
\widehat{C_{\lambda}^{a / q}, 1}(\xi)=\sum_{\ell \in \mathbb{Z}^{\mathrm{d}}} \mathrm{G}(\mathrm{a}, \ell, \mathrm{q}) \widetilde{\psi}_{\mathrm{q}}(\xi-\ell / \mathrm{q}) \widetilde{\psi}_{\lambda \mathrm{Q} / \mathrm{N}}(\xi-\ell / \mathrm{q}) \widetilde{\mathrm{d \sigma}}_{\lambda}(\xi-\ell / \mathrm{q}) .
$$

Above, we have adjusted the cutoff around each point $\ell / q \in \mathbb{T}^{d}$.

Small Denominators: The $\ell^{2}$ Part. We complete the construction of the term in $M_{2}$, (2.6), by showing that

$$
\left\|\sup _{N^{1+\varepsilon} \leq \lambda \leq \ell(E)}\left|C_{\lambda}^{a / q, 2} f\right|\right\|_{2} \lesssim q^{-1} N^{-\frac{d-2}{2}}\|f\|_{2} .
$$

It follows that

$$
\left\|\sum_{1 \leq q \leq N^{1}+\epsilon} \sum_{a \in \mathbb{Z}_{q}^{\times}} e_{q}\left(-\lambda^{2} a\right) C_{\tau}^{a / q, 2} f\right\|_{2} \lesssim N^{-\frac{d-4}{2}+\epsilon}\|f\|_{2} .
$$

This is the third and final contribution to $\mathrm{M}_{2}$. We remark that the proof detailed below is a quantitative variant of the proof of Magyar, Stein and Wainger's inequality (2.14). The inequality $(2.16)$ is $[6,(2.14)]$, but we include details here.

Let $m$ be a smooth function supported on $[-1 / 2,1 / 2]^{d}$, and let $T_{m}$ be the corresponding multiplier operator, either on $\mathbb{Z}^{\mathrm{d}}$ or $\mathbb{R}^{\mathrm{d}}$, with the notation indicating in which setting we are considering the multiplier. 
This is a factorization argument from Magyar, Stein and Wainger [13, pg. 200]. Using the notation of (2.23) to define $M_{\psi_{\mathrm{Q} / 2}}$ below, for $\mathrm{Q} \leq \mathrm{q} \leq 2 \mathrm{Q}$, we have

$$
\begin{aligned}
\widehat{C_{\lambda}^{\mathrm{a} / \mathrm{q}, 2}}(\xi) & =M_{\psi_{\mathrm{Q} / 2}, \mathrm{q}}(\xi) \cdot \sum_{\ell \in \mathbb{Z}^{\mathrm{d}}} \widetilde{\psi}_{\mathrm{q}}(\xi-\ell / \mathrm{q})\left(1-\widetilde{\psi}_{\lambda \mathrm{Q} / \mathrm{N}}(\xi-\ell / \mathrm{q})\right) \widetilde{\mathrm{d} \sigma_{\lambda}}(\xi-\ell / \mathrm{q}) \\
& :=\widehat{M_{\psi_{\mathrm{q} / 2}, \mathrm{q}}}(\xi) \cdot \widehat{C_{\lambda}^{\mathrm{a} / \mathrm{q}, 3}}(\xi) .
\end{aligned}
$$

That is, the operator in question factors as $C_{\lambda}^{a / q, 2}=C_{\lambda}^{a / q, 3} \circ M_{\psi_{Q / 2}, q}$. Notice that by the Gauss sum estimate, we have

$$
\left\|M_{\psi_{Q} / 2, q}\right\|_{2 \rightarrow 2} \lesssim Q^{-\frac{d}{2}}
$$

In controlling the supremum, we need only consider the supremum over $C_{\lambda}^{a / q, 3}$. The transference lemma [13, Cor. 2.1] allows us to estimate this supremum on $\mathrm{L}^{2}\left(\mathbb{R}^{\mathrm{d}}\right)$. We have

$$
\left\|C_{\tau}^{\mathrm{a} / \mathrm{q}, 3}\right\|_{\ell^{2}\left(\mathbb{Z}^{\mathrm{d}}\right) \rightarrow \ell^{2}\left(\mathbb{Z}^{\mathrm{d}}\right)} \lesssim\left\|\sup _{\lambda>0}\left|\Psi_{\lambda, \mathrm{q}} \cdot\right|\right\|_{\mathrm{L}^{2}\left(\mathbb{R}^{\mathrm{d}}\right) \rightarrow \mathrm{L}^{2}\left(\mathbb{R}^{\mathrm{d}}\right)},
$$

where $\widetilde{\Psi}_{\lambda, q}=\widetilde{\psi}_{q}\left(1-\widetilde{\psi}_{\lambda \mathrm{Q} / \mathrm{N}}\right) \widetilde{\mathrm{d}}_{\lambda}$. To estimate this last norm on $\mathrm{L}^{2}\left(\mathbb{R}^{\mathrm{d}}\right)$, we use this Lemma of Bourgain.

Lemma 2.20. [2, Prop. 2] Let $m$ be a smooth function on $\mathbb{R}^{\mathrm{d}}$. We have

$$
\left\|\sup _{r>0}\left|T_{m(r \cdot)} \cdot\right|\right\|_{2} \lesssim \sum_{j \in \mathbb{Z}} \alpha_{j}^{1 / 2}\left(\alpha_{j}^{1 / 2}+\beta_{j}^{1 / 2}\right)
$$

where

$$
\alpha_{j}=\left\|\mathbf{1}_{2^{j} \leq|\xi| \leq 2^{j+1}} m(\xi)\right\|_{\infty} \quad \text { and } \quad \beta_{j}=\left\|1_{2^{j} \leq|\xi| \leq 2^{j+1}} \nabla m(\xi) \cdot \xi\right\|_{\infty} .
$$

We bound the right side of (2.19). Composition with $T_{\psi_{q}}$ is uniformly bounded on $\mathrm{L}^{2}$. The multiplier in question is then, $\mathrm{m}(\xi)=\left(1-\widetilde{\psi}_{\mathrm{Q} / \mathrm{N}}\right)(\xi) \widetilde{d \sigma_{1}}(\xi)$. This is identically zero for $|\xi| \lesssim N / Q$. That means that for the terms in (2.21), we need only consider $2^{j} \gtrsim N / Q \geq 100$. Recall the standard stationary phase estimate below.

$$
\left|\nabla \widetilde{\mathrm{d} \sigma_{1}}(\xi)\right|+\left|\widetilde{\mathrm{d} \sigma_{1}}(\xi)\right| \lesssim|\xi|^{-\frac{\mathrm{d}-1}{2}} .
$$

Hence, the bound for our multiplier is

$$
\begin{aligned}
\left\|\sup _{\lambda>0} \mid \Psi_{\lambda, q} \cdot\right\| \|_{L^{2}\left(\mathbb{R}^{\mathrm{d}}\right) \rightarrow \mathrm{L}^{2}\left(\mathbb{R}^{\mathrm{d}}\right)} & \lesssim \sum_{j: 2^{j} \geq \mathrm{N} / \mathrm{Q}} 2^{-\mathrm{j} \frac{\mathrm{d}-1}{2}} \cdot 2^{-\mathrm{j} \frac{\mathrm{d}-3}{2}} \\
& \lesssim(\mathrm{Q} / \mathrm{N})^{\frac{\mathrm{d}-2}{2}} .
\end{aligned}
$$

This estimate combined with (2.18) and (2.19) complete the proof of (2.16). 
Small Denominators: The Sparse Part. Recalling the notation from (2.15), we turn to

$$
M_{1,2} f=\sum_{1 \leq q \leq N} \sum_{a \in \mathbb{Z}_{q}^{x}} e_{q}\left(-\lambda^{2} a\right) C_{\tau}^{a / q, 1} f
$$

and show that this term is as in (2.7). This is the term in which the absorbing term $\frac{1}{2} A_{\tau} f$ in (2.7) arises. It is also the core of the proof.

Define

$$
M_{1, Q} f=\sum_{Q \leq q<2 Q} \sum_{a \in \mathbb{Z}_{q}^{x}} e_{q}\left(-\lambda^{2} a\right) C_{\tau}^{a / q, 1} f, \quad 1 \leq Q \leq N / 2,
$$

Above, and below, we will treat $M_{1, Q}$ as an operator, as we have yet to tease out some of its additional properties. The main estimate to prove is

$$
\begin{aligned}
M_{1, Q} f & \leq \bar{M}_{1, Q} f+N^{-\epsilon} A_{\tau} f, \\
|E|^{-1}\left\langle\bar{M}_{1, Q} f, g\right\rangle & \lesssim N^{1+\epsilon / 2}\langle f\rangle_{E}^{\frac{1}{\bar{p}+\epsilon}}\langle g\rangle_{E}^{\frac{1}{\bar{q}+\epsilon}} .
\end{aligned}
$$

This summed over dyadic $1 \leq \mathrm{Q} \leq \mathrm{N} / 2$ to complete the proof of the absorption inequality (2.7). This step requires that $N$ be sufficiently large, $N>\kappa^{1 / \epsilon}$, but that is sufficient for our purposes.

We need the estimate (2.4) on $\mathbb{R}^{\mathrm{d}}$. We also need kernel estimates for the operators $M_{1, Q}$, and for that we require this preparation, which has been noted before [13], [6, pg. 1415]. For a function $\zeta$ with $\widetilde{\zeta}$ supported on $[-1,1]^{\text {d }}$, define a family of Fourier multipliers by

$$
\widehat{M_{\zeta, q}}(\xi)=\sum_{\ell \in \mathbb{Z}_{q}^{\mathrm{d}}} \mathrm{G}(\mathrm{a} / \mathrm{q}, \ell) \widetilde{\zeta}(\xi-\ell / \mathrm{q}) .
$$

By inspection, the Gauss sum map $\ell \mapsto G(a / q, \ell)$ is the Fourier transform of $e_{q}\left(|x|^{2} a\right)$ as a function on $\mathbb{Z}_{\mathrm{q}}^{\mathrm{d}}$. From this, and a routine computation, it follows that

$$
M_{\zeta, q}(x)=e_{q}\left(a|x|^{2}\right) \zeta(x)
$$

(Here we identify the kernel of the convolution operator, and the operator itself.)

It follows that the kernel of $M_{1, Q} f$ is

$$
\begin{aligned}
M_{1, Q}(n) & =\psi_{\tau Q / N} * d \sigma_{\tau}(n) \sum_{Q \leq q \leq 2 Q} \sum_{a \in \mathbb{Z}_{q}^{x}} e_{q}\left(a\left(|n|^{2}-\lambda^{2}\right)\right) \\
& =P_{Q, \tau}(n) \cdot C_{Q}\left(|n|^{2}-\lambda^{2}\right) .
\end{aligned}
$$

Note that $\mathrm{P}_{\mathrm{Q}, \tau}$ is a maximal average over annuli of outer radius $\tau$, and width about $\tau \mathrm{Q} / \mathrm{N}<\tau$. The second term above is related to Ramanujan sums, defined by

$$
c_{\mathrm{q}}(\mathrm{m})=\sum_{\mathrm{a} \in \mathbb{Z}_{\mathrm{q}}^{\times}} e_{\mathrm{q}}(\mathrm{am}), \quad \mathrm{m} \in \mathbb{Z},
$$


so that in (2.24), $\mathrm{C}_{\mathrm{Q}}=\sum_{\mathrm{Q} \leq \mathrm{q} \leq 2 \mathrm{Q}} \mathrm{C}_{\mathrm{q}}$. Ramanujan sums satisfy very good cancellation properties. The properties we will need are summarized in

Lemma 2.25. These two estimates hold, for any $k \in \mathbb{N}$, and $\epsilon>0$,

(1) For any $\mathrm{Q}$ and $\mathrm{n},\left|\mathrm{C}_{\mathrm{Q}}(\mathrm{n})\right| \leq \mathrm{Q}^{2}$.

(2) There holds

$$
\max _{0<m \leq Q^{k}}\left|C_{Q}(m)\right| \lesssim Q^{1+\epsilon}
$$

(3) For $M>Q^{k}$,

$$
\left[\frac{1}{M} \sum_{m \leq M}\left|C_{Q}(m)\right|^{k}\right]^{1 / k} \lesssim Q^{1+\epsilon}
$$

The implied constants depend upon $\mathrm{k}$ and $\epsilon$.

Proof. The first estimate is trivial, but we include it for the sake of clarity. Note that $\mathrm{C}_{\mathrm{Q}}(0) \simeq \mathrm{Q}^{2}$, which fact will arise in the absorption argument below.

An argument for the second inequality (2.26) begins with the inequality $\left|c_{q}(m)\right| \leq$ $(q, m)$, for $m>0$. This can be checked by inspection if $q$ is a power of a prime. The general case follows as both sides are multiplicative functions.

Then, of course we have for any $1 \leq \mathrm{d} \leq \mathrm{q}$,

$$
\sum_{k: d k \leq Q} d \leq Q
$$

It follows that

$$
\sum_{\mathrm{q} \leq \mathrm{Q}}(\mathrm{q}, \mathrm{m}) \leq \mathrm{Q} \sum_{\mathrm{d} \leq \mathrm{q}: \mathrm{d} \mid \mathrm{m}} 1=\mathrm{Q} \delta(\mathrm{m} ; \mathrm{Q}),
$$

where $\delta(\mathrm{m} ; \mathrm{Q})$ is the number of divisors of $\mathrm{m}$ that are less than or equal to $\mathrm{Q}$. But, $\mathrm{m} \leq \mathrm{Q}^{\mathrm{k}}$, so by a well known logarithmic type estimate for the divisor function, we have (2.26).

The third property is harder. It is due to Bourgain [1]. There are proofs in [10, Lemma 2.13] and [8, Lemma 5].

A Tertiary Decomposition. The preparations are finished. It remains to prove (2.22), and this argument is indicated in Figure 4. There are three cases, namely

(1) $\mathrm{Q}<\mathrm{N}^{1 / 2}$.

(2) $N Q^{k_{1}-1}<\tau$, where $k_{1}=k_{1}(\bar{p}, \bar{q})$.

(3) $N^{1 / 2} \leq Q$ and $\tau \leq N Q^{k_{1}-1}$, implying $N<\tau<Q^{k_{2}}$, where $k_{2}=k_{2}(\bar{p}, \bar{q})$. 


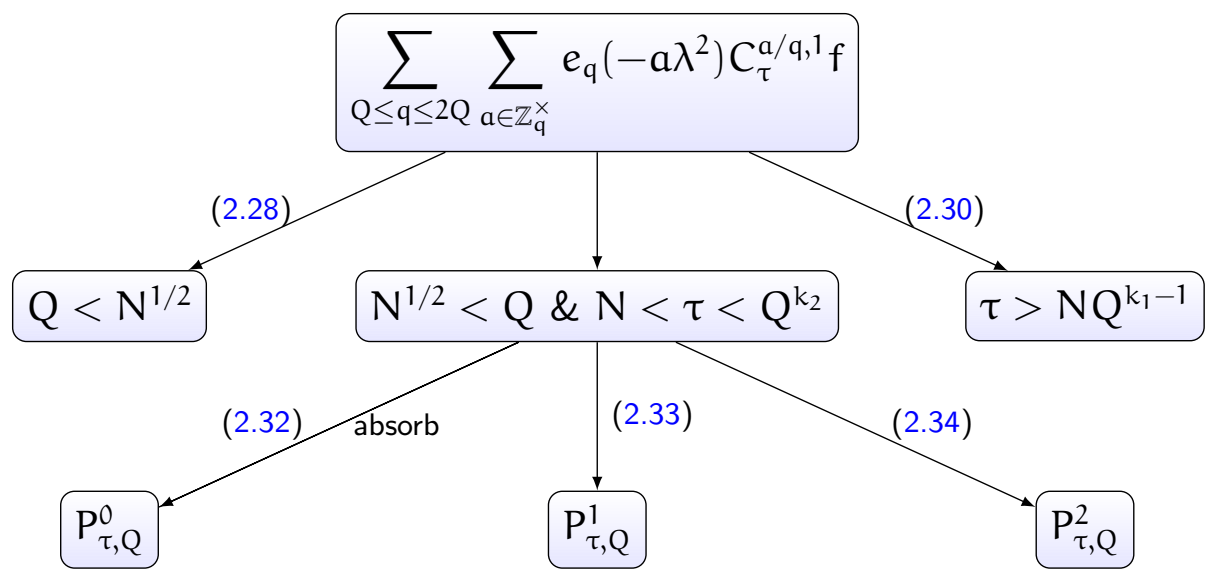

FiguRE 4. The flow of the proof of (2.22). The integers $k_{1}$ and $k_{2}$ are large, and a function of $\epsilon, \bar{p}$ and $\bar{q}$. The first level of the decomposition is motivated by the estimates for Ramanujan sums in Lemma 2.25. The second level of the diagram is associated with the Ramanujan estimate (2.26). It requires a further decomposition of the kernel $P_{\tau, Q}$ in (2.24).

We treat these cases in order, with the core case being the last one.

The first, and easiest case, concerns $Q<N^{1 / 2}$. Using the trivial bound $\left|c_{q}(n)\right| \leq q$, and using (2.24) we have

$$
\left|M_{1, Q} f\right|<N \cdot P_{Q, \tau} f \text {. }
$$

It then follows from the continuous sparse bound (2.4) that we have

$$
|E|^{-1}\left\langle M_{1, Q} f, g\right\rangle \lesssim N\langle f\rangle_{E}^{1 / \bar{p}}\langle g\rangle^{1 / \bar{q}}
$$

This is as required in (2.22).

The second case we restrict to the case that $\tau>N^{k_{1}-1}$, for a sufficiently large integer $k_{1}$ that is a function of $(\bar{p}, \bar{q})$. This case does not have an absorbing term.

Dominate, using Hölder's inequality with $\ell^{k_{1}}-\ell^{k_{1}^{\prime}}$ duality,

$$
\begin{aligned}
\left|M_{1, Q} f(n)\right| & \lesssim\left[P_{Q, \tau} * f(n)\right]^{1 / k_{1}^{\prime}}\left[\sum_{x \in \mathbb{Z}^{d}}\left|C_{Q}\left(|x|^{2}-\lambda^{2}\right)\right|^{k_{1}} P_{Q, \tau}(x)\right]^{1 / k_{1}} \\
& \lesssim Q^{1+\epsilon / 2} P_{Q, \tau} * f(n)^{1 / k_{1}^{\prime}} .
\end{aligned}
$$

Recall that $f$ is an indicator function. Notice that we are using a bound on the Ramanujan sums that follows from (2.27). Recall that $\mathrm{P}_{\mathrm{Q}, \tau}$ is an average over an annulus around the sphere of radius $\lambda$, of width $\tau Q / N$. In particular, the width is greater that $Q^{k_{1}}$, by assumption that $\tau>\mathrm{NQ}^{\mathrm{k}_{1}-1}$.

But, then, we are free to conclude our statement, since $(1 / \bar{p}, 1 / \bar{q})$ are in the interior of $\mathbf{R}_{d}$, we have, for $k_{1}$ sufficiently large, so that $k_{1}^{\prime}$ is sufficiently close to 1 , as required 
in (2.22),

$$
\begin{aligned}
|E|^{-1}\left\langle\left|M_{1} f\right|, g\right\rangle & \lesssim Q^{1+\epsilon}|E|^{-1}\left\langle\left[P_{Q, \tau} * f\right]^{1 / k_{1}^{\prime}}, g\right\rangle \\
& \lesssim N\left[|E|^{-1}\left\langle P_{Q, \tau} * f, g\right\rangle\right]^{1 / k_{1}^{\prime}} \\
& \lesssim\langle f\rangle_{E}^{\frac{1}{\bar{p} k_{1}^{\prime}}}\langle g\rangle_{E}^{\frac{1}{\bar{q} k_{1}^{\prime}}} .
\end{aligned}
$$

Here, we use (2.29) and then the real variable inequality (2.4), which we can do if $k_{1}$ is sufficiently large, so that $\left(k_{1}^{\prime} \bar{p}, k_{1}^{\prime} \bar{q}\right) \in \mathbf{R}_{d}$. This is our second application of (2.4).

We turn to third case of $N<\tau<Q^{k_{2}}$. A final, fourth decomposition of $P_{\tau, Q}$ is needed, and the absorption argument appears. Let $\mathbb{S}_{\lambda}=\left\{n \in \mathbb{Z}^{\mathrm{d}}:|\mathrm{n}|=\lambda\right\}$ be the integer sphere of radius $\lambda$, and set

$$
\begin{aligned}
P_{\tau, Q} & =\sum_{j=0}^{2} P_{\tau, Q}^{j}, \\
\text { where } & P_{\tau, Q}^{0}(n)=P_{\tau, Q}(n) 1_{\mathbb{S}_{\tau}}(n), \\
\text { and } \quad P_{\tau, Q}^{1}(n) & =P_{\tau, Q}(n) 1_{0<\operatorname{dist}\left(n, \mathbb{S}_{\tau}\right)<\tau Q^{1+\epsilon} / N},
\end{aligned}
$$

and $\mathrm{P}_{\tau, \mathrm{Q}}^{2}$ is then defined. The term $\mathrm{P}_{\tau, \mathrm{Q}}^{0}$ is a multiple of the average over the integer sphere of radius $\lambda$, and the term $P_{\tau, Q}^{1}$ is just that part of $P_{\tau, Q}$ that is close to, but not equal to, the sphere of radius $\mathbb{S}_{\tau}$.

Let us detail the absorbing term. Note that $C_{Q}(0) \simeq Q^{2}$. Indeed, we have to single out this case as there is no cancellation in the Ramanujan sum, when the argument is zero. Using the definition (2.31), we have

$$
\begin{aligned}
\left|P_{\tau, Q}^{0}(n) \cdot C_{Q}\left(|n|^{2}-\tau^{2}\right)\right| & \lesssim Q^{2} \frac{N}{Q \tau^{d}} \mathbf{1}_{\mathbb{S}_{\tau}}(n) \\
& \lesssim \frac{N Q}{\tau^{2}} \cdot \tau^{2-d} \mathbf{1}_{\mathbb{S}_{\tau}}(n) \lesssim N^{-2 \epsilon} \cdot \tau^{2-d} \mathbf{1}_{\mathbb{S}_{\tau}}(n) .
\end{aligned}
$$

This is as required in (2.7) and (2.22).

The inequality (2.26) on the Ramanujan sums applies in the analysis of $P_{\tau, Q}^{1}$, due to our assumptions $\mathrm{Q}<\tau<\mathrm{Q}^{\mathrm{k}_{2}}$. It shows that

$$
\left|P_{Q, \tau}^{1}(n) \cdot C_{Q}\left(|n|^{2}-\tau^{2}\right)\right| \lesssim Q^{1+\epsilon} \frac{N}{\tau^{d} Q} 1_{0<\operatorname{dist}\left(n, \mathbb{S}_{\tau}\right)<\tau Q^{1+\epsilon} / N}
$$

Keeping normalizations in mind, it follows from our real variable sparse inequality (2.4) that

$$
|E|^{-1}\left\langle P_{Q, \tau}^{1} * f, g\right\rangle \lesssim Q^{1+2 \epsilon}\langle f\rangle_{E}^{1 / \bar{p}}\langle g\rangle_{E}^{1 / \bar{q}}
$$

This is as required in (2.22). 
The last term is $\mathrm{P}_{\mathrm{Q}, \tau}^{2}$. As noted, $\left|\mathrm{C}_{\mathrm{q}}(\mathrm{m})\right| \leq \mathrm{Q}^{2}$. The condition $\tau<\mathrm{Q}^{\mathrm{k}_{2}}$, and simple Schwartz tail considerations then show that

$$
\left|P_{Q, \tau}^{2}(n) \cdot C_{Q}\left(|n|^{2}-\tau^{2}\right)\right| \lesssim \frac{Q}{\tau^{d}}[1+|n| / \tau]^{-2 d}
$$

Since $\tau$ is an admissible stopping time, it follows that

$$
\left|P_{Q, \tau}^{1} * f\right| \lesssim\langle f\rangle_{E} \text {. }
$$

This completes the proof of (2.22).

\section{The Endpoint Sparse Bound}

We need this definition. Given cube $\mathrm{E}$, we say that collection $\mathcal{Q}_{\mathrm{E}}$ of subcubes $\mathrm{Q} \subset \mathrm{E}$ are pre-sparse if the cubes $\left\{\frac{1}{3} \mathrm{Q}: \mathrm{Q} \in \mathcal{Q}_{\mathrm{E}}\right\}$ are pairwise disjoint. Associated to to a pre-sparse collection $\mathcal{Q}_{\mathrm{E}}$ are a family of stopping times. We say that $\tau$ is $\mathcal{Q}_{\mathrm{E}}$ admissible (or just admissible) if

$$
\ell(E) \geq \tau_{\mathcal{Q}_{\mathrm{E}}}(x)=\tau(x) \geq \max \left\{1, \ell(\mathrm{Q}) \mathbf{1}_{\frac{1}{3} \mathrm{Q}}: \mathrm{Q} \in \mathcal{Q}_{\mathrm{E}}\right\}, \quad x \in \mathrm{E} .
$$

The relevant Lemma is this.

Lemma 3.1. For $\mathrm{f}=\mathbf{1}_{\mathrm{F}}$ supported on cube $3 \mathrm{E}$, there is a pre-sparse collection $\mathcal{Q}_{\mathrm{E}}$ so that for all $\mathcal{Q}_{\mathrm{E}}$ admissible $\tau=\tau(\chi)$, and all $\mathrm{g}=\mathbf{1}_{\mathrm{G}}$ supported on $\mathrm{E}$, we have

$$
\left\langle A_{\tau} f, g\right\rangle \lesssim\langle f\rangle_{3 E, \frac{d}{d-2}}\langle g\rangle_{E, \frac{d}{d-2}}|E| \text {. }
$$

The Lemma follows from this: For integers $N>1$, there is a decomposition

$$
\begin{aligned}
A_{\tau} f & \leq M_{1}+M_{2}, \\
\left\langle M_{1}, g\right\rangle & \lesssim N^{2}\langle f\rangle_{3 E, 1}\langle g\rangle_{E, 1}|E|, \\
\left\langle M_{2}, g\right\rangle & \lesssim N^{-\frac{d-4}{2}}\langle f\rangle_{3 E, 2}\langle g\rangle_{E, 2}|E| .
\end{aligned}
$$

Recalling that $f=\mathbf{1}_{\mathrm{F}}$ and $\mathrm{g}=\mathbf{1}_{\mathrm{G}}$, the right sides above are comparable for $\mathrm{N} \simeq$ $\left[\langle\mathbf{f}\rangle_{3 \mathrm{E}, 1}\langle\mathbf{g}\rangle_{\mathrm{E}, 1}\right]^{-\frac{1}{\mathrm{~d}}}$, and this proves (3.2).

It remains to prove (3.3)-(3.5). Our proof will be much shorter, because we do not need to compare to the very rough continuous spherical averages, but to averages over balls. (In particular, we will not need any subtle facts about Ramanujan sums.) A guide to the argument is in Figure 5. The decomposition has several elements. The first begins with the trivial bound $A_{\lambda} f(x) \lesssim \lambda^{2} B_{\lambda} * f(x)$, where $B_{\lambda}$ is the average of over a ball of radius $\lambda$. Our first contribution to $M_{1}$ is,

$$
M_{1,1}=\mathbf{1}_{\tau(x) \leq 100 N} A_{\tau} f,
$$

which is pointwise bounded by $C N^{2}\langle f\rangle_{3 E, 1}$, by choice of $\mathcal{Q}_{E}$. Thus (3.4) holds for this term. Below, we are free to assume that $\tau \geq 100 \mathrm{~N}$. 


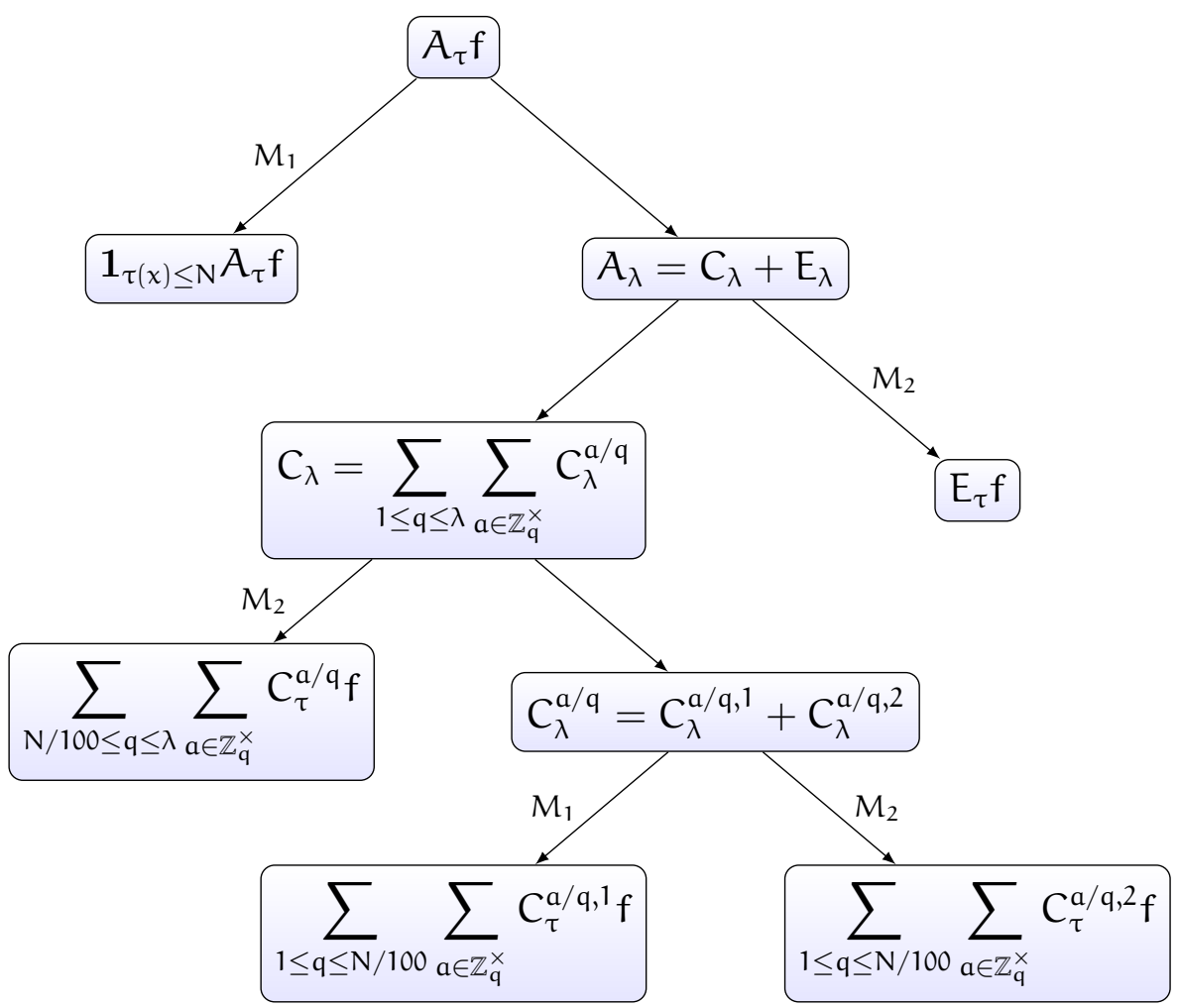

Figure 5. The flow of the proof of (3.3)-(3.5). The notation is similar to Figure 3.

Recall the decomposition of $A_{\lambda} f$, beginning with (2.9). Our first contribution to $M_{2}$ is $M_{2,1}=\left|E_{\tau} f\right|$. This satisfies (3.5) by (2.12).

It remains to bound $C_{\tau} f$ as defined in (2.10). This requires further contributions to $M_{1}$ and $\mathrm{M}_{2}$. Apply (2.14) to see that this term obeys (3.5).

$$
M_{2,2}=\sum_{N / 100 \leq q \leq \lambda} \sum_{a \in \mathbb{Z}_{q}^{\times}}\left|C_{\tau}^{a / q} f\right|
$$

The remaining terms are

$$
\sum_{1 \leq q \leq N / 100} \sum_{a \in \mathbb{Z}_{q}^{\times}} e_{q}\left(-\lambda^{2} a\right) C_{\tau}^{a / q} f
$$

Control will consist of an additional contribution to $M_{1}$ and $M_{2}$. 
Write $C_{\lambda}^{a / q}=C_{\lambda}^{a / q, 1}+C_{\lambda}^{a / q, 2}$, where a different cut-off in frequency is inserted.

$$
\begin{aligned}
\widehat{C_{\lambda}^{a / q}, 1}(\xi) & =\sum_{\ell \in \mathbb{Z}^{d}} G(a / q, \ell) \widetilde{\psi}_{q}(\xi-\ell / q) \widetilde{\psi}_{\lambda q / N}(\xi-\ell / q) \widetilde{d \sigma_{\lambda}}(\xi-\ell / q) \\
& =\sum_{\ell \in \mathbb{Z}^{d}} G(a / q, \ell) \widetilde{\psi}_{\lambda q / N}(\xi-\ell / q) \widetilde{d \sigma_{\lambda}}(\xi-\ell / q) .
\end{aligned}
$$

This follows from the definition of $\psi$ in (2.11). This is slightly different from (2.15), in particular, the term below satisfies (3.5), just as in the previous section.

$$
M_{2,3}=\left|\sum_{1 \leq q \leq N / 100} \sum_{a \in \mathbb{Z}_{q}^{\times}} e_{q}\left(-\lambda^{2} a\right) C_{\tau}^{a / q, 2} f\right| .
$$

We claim that

$$
\left\|\sum_{1 \leq \mathrm{q} \leq \mathrm{N} / 100} \sum_{\mathrm{a} \in \mathbb{Z}_{\mathrm{q}}^{\times}} e_{\mathrm{q}}\left(-\lambda^{2} a\right) \mathrm{C}_{\tau}^{\mathrm{a} / \mathrm{q}, 1} \mathrm{f}\right\|_{\infty} \lesssim N^{2}\langle f\rangle_{E, 1} .
$$

That is, the term on the left is our second and final contribution to $M_{1}$, as in (3.4).

Recalling (2.23) and (2.24), we have

$$
\left|C_{\lambda}^{\mathrm{a} / \mathrm{q}, 1}(\mathrm{n})\right| \lesssim \mathrm{d} \sigma_{\lambda} * \psi_{\lambda \mathrm{q} / \mathrm{N}}(\mathrm{n})
$$

The convolution is with $d \sigma_{\lambda}$ and $\psi_{\lambda q / N}$, which is a bump function of integral one, supported on scale $\mathrm{N} / \lambda \mathrm{q}$, which is much smaller than $\lambda$. As a consequence, we have

$$
\left|C_{\lambda}^{\mathrm{a} / \mathrm{q}, 1}(\mathrm{n})\right| \lesssim \frac{\mathrm{N}}{\mathrm{q}} \cdot \lambda^{-\mathrm{d}}[1+|\mathrm{n}| / \lambda]^{-3 \mathrm{~d}} .
$$

This is summed over $1 \leq \mathrm{a}<\mathrm{q} \leq \mathrm{N} / 100$. And, one appeals to the admissibility of the stopping time $\tau$ to complete the proof of (3.6).

\section{Interpolation of Sparse Bounds}

We show that if a sublinear operator satisfies an open range of sparse bounds for indicator sets, then they improve to sparse bounds for functions.

Lemma 4.1. Suppose that a sub-linear operator $T$ satisfies the bound below for $1<$ $\mathrm{p}, \mathrm{q}<\infty$. For a fixed function $\mathrm{f}$ and all $|\mathrm{g}| \leq \mathbf{1}_{\mathrm{G}}$, there is a sparse collection $\mathcal{S}$ so that

$$
|\langle\mathrm{Tf}, \mathrm{g}\rangle| \lesssim \wedge_{\mathcal{S}, \mathrm{p}, \mathrm{q}}\left(\mathrm{f}, \mathbf{1}_{\mathrm{G}}\right)
$$

Then,

$$
|\langle\mathrm{Tf}, \mathrm{g}\rangle| \lesssim \sup _{\mathcal{S}} \Lambda_{\mathcal{S}, \mathrm{p}, \mathrm{q}}(\mathrm{f}, \mathrm{g}), \quad \mathrm{q}<\mathrm{r}<\infty .
$$


Proof. In this proof, we will work on $\mathbb{R}^{\mathrm{d}}$, with the same proof working on $\mathbb{Z}^{\mathrm{d}}$. We will also assume that (1) $\mathrm{T}$ is a positive operator, and (b) all cubes are dyadic. The general case is not much harder than these considerations. Let $\mathrm{f}$, and let $\mathrm{g}$ be a bounded compactly supported function. We will show that there is a sparse collection $\mathcal{S}$ so that

$$
\langle\mathrm{Tf}, \mathrm{g}\rangle \lesssim \sum_{\mathrm{Q} \in \mathcal{S}}\left\langle\mathbf{1}_{\mathrm{F}}\right\rangle_{\mathrm{Q}}^{1 / \mathrm{p}}\langle\mathrm{g}\rangle_{\mathrm{Q}, \mathrm{q}, 1}|\mathrm{Q}|,
$$

where $\langle g\rangle_{\mathrm{Q}, \mathrm{q}, 1}=\left\|\mathrm{g} \mathbf{1}_{\mathrm{Q}}\right\|_{\mathrm{L}^{\mathrm{q}, 1}(\mathrm{~d} x / \mathrm{Q} \mid)}$ is the Lorentz space with normalized measure. Since $\langle\mathrm{g}\rangle_{\mathrm{Q}, \mathrm{q}, 1}<\langle\mathrm{g}\rangle_{\mathrm{Q}, \mathrm{r}}$, for $1<\mathrm{r}<\mathrm{q}$, this completes the proof of the Lemma.

The argument for (4.2) is a level set argument. Thus, write $g \leq \sum_{k \in \mathbb{Z}} 2^{k} \mathbf{1}_{G_{k}}$, for disjoint sets $G_{k}$. Apply the assumed sparse bound for indicators for each pair of sets $\left(F, G_{k}\right)$. We get a sequence of sparse sets $\mathcal{S}_{k}$ so that

$$
\langle\mathrm{Tf}, \mathrm{g}\rangle \lesssim \sum_{\mathrm{k} \in \mathbb{Z}} \Lambda_{\mathcal{S}_{\mathrm{k}}, \mathrm{p}, \mathrm{q}}\left(\mathbf{1}_{\mathrm{F}}, \mathbf{1}_{\mathrm{G}_{\mathrm{k}}}\right)
$$

Let $\mathcal{F}$ be a sequence of stopping cubes for the averages $\langle f\rangle_{\mathrm{Q}, p}$. That is, we choose $\mathcal{F}$ so that for any dyadic cube $\mathrm{Q}$, there is a dyadic cube in $\mathcal{F}$ that contains $\mathrm{Q}$. And setting $\mathrm{Q}^{a}$ to be the minimal such cube in $\mathcal{F}$, we have $\langle f\rangle_{\mathrm{Q}, \mathrm{p}} \lesssim\langle f\rangle_{\mathrm{Q}^{a}, \mathrm{p}}$. The sum in (4.3) is organized according to $\mathcal{F}$. Below, we take $\mathrm{q}<\alpha<\infty$, very close to $\mathrm{q}$. For each $\mathrm{P} \in \mathcal{F}$ we have

$$
\begin{aligned}
\Gamma(\mathrm{P}) & =\sum_{\mathrm{k} \in \mathbb{Z}} 2^{-\mathrm{k}} \sum_{\substack{\mathrm{Q} \in \mathcal{S}_{k} \\
\mathrm{Q}^{\mathrm{a}}=\mathrm{P}}}\left\langle\mathbf{1}_{\mathrm{G}_{\mathrm{k}}}\right\rangle_{\mathrm{Q}}^{1 / \mathrm{q}}|\mathrm{Q}| \\
& \lesssim \sum_{\mathrm{k} \in \mathbb{Z}} 2^{-\mathrm{k}}|\mathrm{P}|^{1 / \alpha^{\prime}}\left[\sum_{\substack{\mathrm{Q} \in \mathcal{S}_{k} \\
\mathrm{Q}^{\mathrm{a}}=\mathrm{P}}}\left\langle\mathbf{1}_{\mathrm{G}_{\mathrm{k}}}\right\rangle_{\mathrm{Q}}^{\alpha / \mathrm{q}}\right]^{1 / \alpha} \\
& \lesssim|\mathrm{P}| \sum_{\mathrm{k} \in \mathbb{Z}} 2^{-\mathrm{k}}\left\langle\mathbf{1}_{\mathrm{G}_{\mathrm{k}}}\right\rangle_{\mathrm{P}}^{1 / \mathrm{q}}=\langle\mathrm{g}\rangle_{\mathrm{L}^{\mathrm{q}, 1}(\mathrm{P})}|\mathrm{P}| .
\end{aligned}
$$

Here, we have used Hölder's inequality, sparseness of the collections $\mathcal{S}_{k}$ and the Carleson embedding inequality. The last inequality is one way to define the $L^{q, 1}$ norm.

And, so we have

$$
(4.3) \lesssim \sum_{\mathrm{P} \in \mathcal{F}}\langle f\rangle_{\mathrm{P}, \mathrm{p}} \Gamma(\mathrm{P}) .
$$

The bound in (4.4) then implies (4.2).

Concerning our Theorem 1.2. For the first assertion, we have proved a restricted weak type inequality for all $(1 / p, 1 / q)$ in the interior of $\mathbf{Z}_{d}$. We see that we can then replace the indicator functions in both coordinates by functions. That is, we have $\langle A\rangle_{p, q}<\infty$ for all $(1 / p, 1 / q)$ in the interior of $\mathbf{Z}_{d}$. 
Concerning the second assertion, we have the restricted weak type inequality at $\left(\frac{d}{d-2}, \frac{d}{d-2}\right)$. We see from the Theorem above that we have this consequence

$$
\langle A f, g\rangle \lesssim \sup _{\mathcal{S}} \sum_{\mathrm{Q} \in \mathcal{S}}\langle f\rangle_{\mathrm{Q}, \frac{\mathrm{d}}{\mathrm{d}-2}, 1}\langle g\rangle_{\mathrm{Q}, \mathrm{q}}|\mathrm{Q}|, \quad \mathrm{q}>\frac{\mathrm{d}}{\mathrm{d}-2} \text {. }
$$

\section{Counterexamples}

We can show this proposition, showing necessary conditions on $(p, q)$ for the sparse bound to hold. The gap between the sufficient conditions for a sparse bound and these necessary conditions is illustrated in Figure 6.

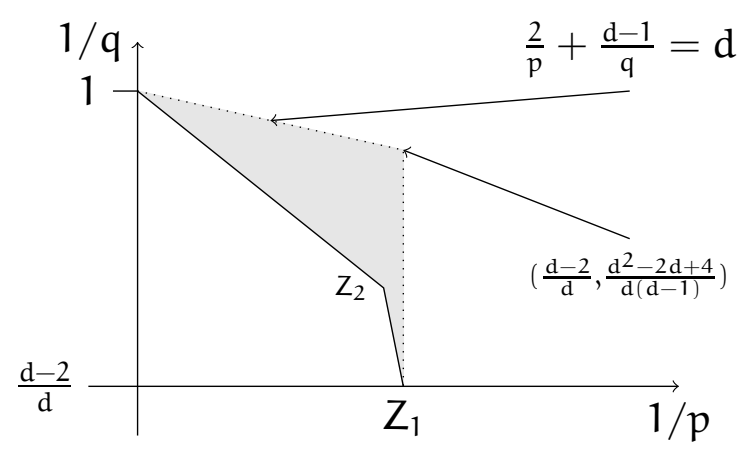

FiguRE 6 . The horizontal line is set at $\frac{1}{q}=\frac{d-2}{d}$, for reasons of clarity. Sparse bounds hold below the solid line from $(0,1)$, to $Z_{2}$ to $Z_{1}=\left(\frac{d-2}{d}, \frac{d-2}{d}\right)$. (Recall that $Z_{2}$ is defined in (1.5).) They cannot hold to the right of $Z_{1}$, nor above the dotted line. The gray area is unresolved.

Proposition 5.1. If the sparse bound $\|A\|_{p, q}<\infty$ holds, we have

$$
\frac{1}{p_{1}} \leq \frac{d-2}{d}, \quad \frac{2}{p}+\frac{d-1}{q} \leq d
$$

Proof. If a $(p, q)$ sparse bound held we would conclude that $A: \ell^{p} \rightarrow \ell^{p, \infty}$. Taking $f=1_{0}$, note that $\operatorname{Af}(x) \simeq(1+|x|)^{2-d}$. The latter function is $\ell \frac{d}{d-2}, \infty$. Hence, $p_{1} \geq \frac{d}{d-2}$ is necessary.

For the second inequality in (5.2), set $\mathbb{S}_{\lambda}=\left\{n \in \mathbb{Z}^{\mathrm{d}}:|n|=\lambda\right\}$. We recall that for $\mathrm{d} \geq 4$, and any odd choice of $\lambda^{2} \in \mathbb{N}$, we have $\left|\mathbb{S}_{\lambda}\right| \simeq \lambda^{\mathrm{d}-2}$. For an odd choice of $\lambda^{2} \in \mathbb{N}$, let $f=\mathbf{1}_{\mathbb{S}_{\lambda}}$, and consider $G=\{A f>c / \lambda\}$. This is the set of $x \in \mathbb{Z}^{\mathrm{d}}$ for which the two spheres $\mathbb{S}_{\lambda}$ and $x+\mathbb{S}_{\mu}$, for some choice of $\mu \simeq \lambda$, have about the expected size. Here, necessarily $G \subset E$, a cube centred at the origin of side length about $\lambda$. 
We claim that $|G| \gtrsim \lambda$. And observe that $\operatorname{Af}(x) \gtrsim \lambda^{-1}$ for $x \in G$. Moreover, from the assumed $(p, q)$ sparse bound,

$$
\begin{aligned}
\lambda^{-1}\left\langle\mathbf{1}_{\mathrm{G}}\right\rangle_{\mathrm{E}} & \lesssim \lambda^{-\mathrm{d}}\langle\text { Af, g }\rangle \\
& \lesssim\langle f\rangle_{\mathrm{E}}^{\frac{1}{p}}\left\langle\mathbf{1}_{\mathrm{G}}\right\rangle_{\mathrm{E}}^{\frac{1}{q}} \lesssim \lambda^{-\frac{2}{p}}\left\langle\mathbf{1}_{\mathrm{G}}\right\rangle_{\mathrm{E}}^{\frac{1}{q}} .
\end{aligned}
$$

For this pair of functions, it is easy to see that the maximal sparse form is the expression on the right. Our lower bound on the size of $\mathrm{G}$ proves the proposition.

Note that since dimension $d \geq 5$, and $\lambda^{2}$ is odd, so there are about $\lambda^{d-3}$ choices of vectors $y=\left(0, y_{2}, \ldots, y_{d}\right) \in \mathbb{S}_{\lambda}$. (We insist on $\lambda^{2}$ being odd to capture the case of $d=5$ here.) Then, if $\left|x_{1}\right| \leq \lambda / 2$, note that

$$
\left\|\left(0, y_{2}, \ldots, y_{d}\right)-\left(x_{1}, 0, \ldots, 0\right)\right\|=\sqrt{\lambda^{2}+x_{1}^{2}}=\lambda^{\prime}
$$

From that, it follows that

$$
A_{\lambda^{\prime}} f\left(x_{1}, 0, \ldots, 0\right) \simeq \lambda^{-1} \text {. }
$$

This shows that $|\mathrm{G}| \gtrsim \lambda$.

Observe that the $\left(\frac{d}{d-2}, \frac{d}{d-2}\right)$ sparse bound and (5.3) imply that $|G| \lesssim \lambda^{\frac{d+4}{2}}$. Our lower bound is certainly not sharp. What is the correct size of $G$ ?

\section{REFERENCES}

[1] J. Bourgain, Fourier transform restriction phenomena for certain lattice subsets and applications to nonlinear evolution equations. I. Schrödinger equations, Geom. Funct. Anal. 3 (1993), no. 2, 107-156. MR1209299

[2] Jean Bourgain, Estimations de certaines fonctions maximales, C. R. Acad. Sci. Paris Sér. I Math. 301 (1985), no. 10, 499-502. MR812567

[3] B. Cook, A note on discrete spherical averages over sparse sequences, ArXiv e-prints (2018), available at 1808.03822.

[4] K. Hughes, The discrete spherical averages over a family of sparse sequences, ArXiv e-prints (September 2016), available at 1609.04313.

[5] _ , $\ell^{p}$-improving for discrete spherical averages, ArXiv e-prints (April 2018), available at 1804.09260.

[6] Alexandru D. Ionescu, An endpoint estimate for the discrete spherical maximal function, Proc. Amer. Math. Soc. 132 (2004), no. 5, 1411-1417. MR2053347

[7] R. Kesler, $\ell^{\mathfrak{p}}\left(\mathbb{Z}^{\mathrm{d}}\right)$-Improving Properties and Sparse Bounds for Discrete Spherical Maximal Averages, ArXiv e-prints (May 2018), available at 1805.09925.

[8],$\ell^{\mathrm{p}}\left(\mathbb{Z}^{\mathrm{d}}\right)$-Improving Properties and Sparse Bounds for Discrete Spherical Maximal Means, Revisited, ArXiv e-prints (September 2018), available at 1809.06468.

[9] R. Kesler and M. T. Lacey, $\ell^{\mathfrak{p}}$-improving inequalities for Discrete Spherical Averages, ArXiv e-prints (April 2018), available at 1804.09845.

[10] R. Kesler, M. T. Lacey, and D. Mena, Lacunary Discrete Spherical Maximal Functions, ArXiv e-prints (October 2018), available at 1810.12344.

[11] M. T. Lacey, Sparse Bounds for Spherical Maximal Functions, ArXiv e-prints (February 2017), available at 1702.08594. 
[12] Sanghyuk Lee, Endpoint estimates for the circular maximal function, Proc. Amer. Math. Soc. 131 (2003), no. 5, 1433-1442. MR1949873

[13] A. Magyar, E. M. Stein, and S. Wainger, Discrete analogues in harmonic analysis: spherical averages, Ann. of Math. (2) 155 (2002), no. 1, 189-208. MR1888798

[14] Akos Magyar, $\mathrm{L}^{\mathrm{p}}$-bounds for spherical maximal operators on $\mathbf{Z}^{\mathrm{n}}$, Rev. Mat. Iberoamericana 13 (1997), no. 2, 307-317. MR1617657

[15] W. Schlag, A generalization of Bourgain's circular maximal theorem, J. Amer. Math. Soc. 10 (1997), no. 1, 103-122. MR1388870

[16] Wilhelm Schlag and Christopher D. Sogge, Local smoothing estimates related to the circular maximal theorem, Math. Res. Lett. 4 (1997), no. 1, 1-15. MR1432805

School of Mathematics, Georgia Institute of Technology, Atlanta GA 30332, USA

E-mail address: robertmkesler@gmail.com

School of Mathematics, Georgia Institute of Technology, Atlanta GA 30332, USA

E-mail address: lacey@math.gatech.edu

Escuela de Matemática, Universidad de Costa Rica, San José, Costa Rica

E-mail address: dario.menaarias@ucr.ac.cr 